\title{
INTEGRAL DOMAINS WHICH ARE ALMOST DEDEKIND
}

\author{
ROBERT W. GILMER, JR.
}

An integral domain $J$ (with unit) will be said to be almost Dedekind if, given any maximal ideal $P$ of $J, J_{P}$ is a Dedekind domain. It follows, in particular, that $J_{P}$ is a discrete valuation ring.

I. S. Cohen, in $[1$, p. 33$]$ gives six necessary and sufficient conditions that a Noetherian domain $J$ be Dedekind. The first of these is that $J_{P}$ be a discrete valuation ring for every maximal ideal $P$ of $J$. This paper gives necessary and sufficient conditions that an integral domain with unit be almost Dedekind and it is shown that these conditions imply each of the other five conditions of Cohen. Finally, we investigate relations between the ideal structure of $D^{\prime}$ and $D$, where $D$ is an almost Dedekind domain with quotient field $K$ and $D \subseteq D^{\prime} \subseteq K$. In particular, we show $D^{\prime}$ is almost Dedekind and that $D^{\prime}$ is the intersection of all quotient rings $D_{P}$ of $D$ where $P$ is a prime ideal of $D$ such that $P D^{\prime} \subset D^{\prime}$. The results obtained also yield another proof to the theorem of MacLane and Schilling [2, p. 781] which asserts that if $D$ is Dedekind, so is $D^{\prime}$.

Theorem 1. The integral domain $D$ with unit is almost Dedekind if and only if

(1) nonzero proper prime ideals of $D$ are maximal, and

(2) primary ideals of $D$ are prime powers.

Proof. We first suppose $D$ is almost Dedekind. (1) follows easily $\left[1\right.$, p. 34]. If $Q$ is primary for the maximal ideal $P$, then $Q D_{P}$ is primary for $P D_{P}$. Since $D_{P}$ is a Dedekind domain, $Q D_{P}=\left(P D_{P}\right)^{k}$ $=P^{k} D_{P}$ for some positive integer $k$. Since $Q$ is primary for $P, Q$ $=Q D_{P} \cap D$. But because $P$ is maximal in $D, P^{k}$ is also primary for $P$ $\left[4\right.$, p. 153]. Thus $P^{k}=P^{k} D_{P} \cap D$ so that $Q=P^{k}$ and one half of our proof is complete.

We now assume that (1) and (2) hold in $D$. If then $P$ is a nonzero proper prime ideal of $D, P$ is minimal so that $P D_{P}$ is the unique nonzero proper prime ideal of $D_{P}$. If $V$ is any nonzero proper ideal of $D_{P}$, then $\sqrt{ } V=P D_{P}$, and thus, $V$ is primary for $P D_{P}$. Hence $Q=V \cap D$ is primary for $P$ in $D$. By hypothesis, $Q=P^{k}$ for some $k$. Therefore, $V=(V \cap D) D_{P}=Q D_{P}=\left(P D_{P}\right)^{k}$. Consequently, every ideal of $D_{P}$ is a prime power, $D_{P}$ is Dedekind, and $D$ is almost Dedekind.

Received by the editors April 18, 1962 and, in revised form, May 3, 1963. 
Corollary 1. If the integral domain $D$ is almost Dedekind and if $A$ is a proper ideal of $D$, then $\bigcap_{k=1}^{\infty} A^{k}=(0)$.

Proof. We let $P$ be a proper prime ideal of $D$ containing $A$. Since $D_{P}$ is Dedekind, $\cap_{k=1}^{\infty} P^{k} D_{P}=(0)$. Moreover, $A^{k} \subseteq P^{k} \subseteq P^{k} D_{P}$ so that $\bigcap_{k=1}^{\infty} A^{k}=(0)$.

In $[3$, p. 426], N. Nakano has given an example of a non-Noetherian integral domain $W$ with unit in which (1) and (2) of Theorem 1 hold. $W$ is therefore an example of an almost Dedekind domain which is not Dedekind.

From Theorem 1, we easily deduce that an integral domain $J$ with unit which is almost Dedekind satisfies the third and fifth conditions considered by Cohen $[1$, p. 33].

Corollary 2. If $P$ is a prime ideal of $J$, there is no ideal properly between $P$ and $P^{2}$.

COROLlARY 3. The primary ideals belonging to a prime ideal of $J$ are totally ordered by inclusion.

In addition, Cohen noted that such a domain $J$ has the following three properties [1, p. 34].

(a) $J$ is integrally closed. (This is true whether integral closure is defined in terms of modules or in terms of roots of monic polynomials.)

(b) For any three ideals $A, B, C$ of $J, A \cap(B+C)=(A \cap B)$ $+(A \cap C)$.

(c) For any three ideals $A, B, C$ of $J, A:(B \cap C)=(A: B)+(A: C)$.

If $D$ is any integral domain with unit, if $A$ is an ideal of $D$, if $\left\{M_{\lambda}\right\}_{\lambda \in \Delta}$ is the collection of maximal ideals of $D$, and if for $\lambda \in \Lambda$, $A_{\lambda}=A D_{M_{\lambda}} \cap D$, then $A=\cap A_{\lambda}[5$, p. 94$]$.

If now $D$ is almost Dedekind and $A \neq(0)$, then for $A \nsubseteq M_{\lambda}, A D_{M_{\lambda}}$ $=D_{M_{\lambda}}$ and $A_{\lambda}=D=\left(M_{\lambda}\right)^{0}$. But if $A \subseteq M_{\lambda}, A D_{M_{\lambda}}$ is primary for $M_{\lambda} D_{M_{\lambda}}$ so that $A D_{M_{\lambda}}=M_{\lambda}^{k} D_{M_{\lambda}}$ and $A_{\lambda}=M_{\lambda}^{k}$ for some integer $k$. If $\delta$ is the collection of nonzero ideals of $D$, then for $\lambda \in \Lambda$, we denote by $f_{\lambda}$ the function from $S$ into $Z$ such that for $A \in S, f_{\lambda}(A)$ is the smallest nonnegative integer $k$ such that $A_{\lambda}=M_{\lambda}^{k}$. It follows from the preceding paragraph that

$$
A=\bigcap_{\lambda \in \Lambda} M_{\lambda}^{f_{\lambda}(A)}
$$

for each $A \in \mathcal{S}$. Further, if $D^{*}=D-\{0\}$, then for each $\lambda \in \Lambda$ we may define $V_{\lambda}: D^{*} \rightarrow Z$ by $V_{\lambda}(x)=f_{\lambda}((x))$. Theorem 2 gives useful information concerning the functions $V_{\lambda}$ and $f_{\lambda}$. 
Theorem 2. Let $D, D^{*}, A, \Lambda, \lambda, f_{\lambda}$, and $V_{\lambda}$ be as in the preceding paragraph.

(i) There exists an integer $k \geqq 0$ such that $A \subseteq M_{\lambda}^{k}, A \Phi M_{\lambda}^{k+1}$. Further, $k=f_{\lambda}(A)$.

(ii) For $x, y \in D^{*}, \quad V_{\lambda}(x y)=V_{\lambda}(x)+V_{\lambda}(y)$ and $V_{\lambda}(x+y)$ $\leqq \min \left\{V_{\lambda}(x), V_{\lambda}(y)\right\}$. Therefore, $V_{\lambda}$ determines in a canonical way $a$ valuation $s_{\lambda}$ of the quotient field $K$ of $D[5, p .37] . D_{M_{\lambda}}$ is the valuation ring of $s_{\lambda}$.

(iii) For $m_{\lambda} \in M_{\lambda}-M_{\lambda}^{2}$ and for $0 \neq \xi \in K$, there exist $u, v \in D-M_{\lambda}$ such that

$$
\xi=u m_{\lambda}^{\delta^{\lambda}(\xi)} / v
$$

Proof. That such an integer $k$ in (i) exists is an immediate consequence of Corollary 1. If now $A \subseteq M_{\lambda}^{t}, A \Phi M_{\lambda}^{t+1}$, then $A D_{M_{\lambda}}=M_{\lambda}^{r} D_{M_{\lambda}}$ for some $r \geqq t$. If $a \in A-M_{\lambda}^{t+1}$, then because $M_{\lambda}^{t+1}=D \cap M_{\lambda}^{t+1} D_{M_{\lambda}}$, $a \in A D_{M_{\lambda}}-M_{\lambda}^{t+1} D_{M_{\lambda}}$. Thus $A D_{M_{\lambda}}=M_{\lambda}^{t} D_{M_{\lambda}}$ and $t=f_{\lambda}(A)$ as asserted.

The statements in the first sentence of (ii) follow easily from the definition of $V_{\lambda}$. Then from (i) it is clear that the valuation ring of $s_{\lambda}$ contains $D_{M_{\lambda}}$. Because $D_{M_{\lambda}}$ is a maximal subring of $K[\mathbf{5}, \mathrm{p} .17]$, $D_{M_{\lambda}}$ is the valuation ring of $s_{\lambda}$.

In view of (i), $s_{\lambda}\left(m_{\lambda}\right)=1$. Thus if $y=\xi / m_{\lambda}^{s_{\lambda}(\xi)}$, then $s_{\lambda}(y)=0$. Hence $y$ is a unit of $D_{M_{\lambda}}$. Because $y \in D_{M_{\lambda}}, y=u / v$ for some $u \in D, v \in D-M_{\lambda}$. Now $s_{\lambda}(v)=0$ so that $s_{\lambda}(u)=0$ also-that is, $u \notin M_{\lambda}$.

Theorem 3. Let $D$ be an integral domain with unit which is almost Dedekind. $D$ is a Dedekind domain if and only if every nonzero proper ideal of $D$ is contained in only finitely many maximal ideals. In particular, an almosl Dedekind domain with only a finite number of maximal ideals is a principal ideal domain.

Proof. We note that if $M_{1}, \cdots, M_{t}$ are distinct maximal ideals of $D$ and if $e_{1}, \cdots, e_{t}$ are positive integers, then the ideals $M_{1}^{e_{1}}, \cdots$, $M_{t}^{e_{t}}$ are pairwise comaximal. Hence $\bigcap_{i=1}^{t_{1}} M_{i}^{e_{i}}=\prod_{i=1}^{t} M_{i}^{e_{i}}$. Our theorem now follows from the paragraph preceding Theorem 2 and from the known theory of Dedekind domains.

In terms of the valuations of the quotient field of an almost Dedekind domain $D$ we can phrase Theorem 3: $D$ is Dedekind if and only if $D$ is a Krull domain [5, p. 82].

We next consider domains between an almost Dedekind domain and its quotient field.

Theorem 4. Suppose $D$ is an almost Dedekind domain, $K$ is the 
quotient field of $D$, and $D^{\prime}$ is a domain such that $D \subseteq D^{\prime} \subseteq K$. We let $\Delta$ be the set of prime ideals $P$ of $D$ such that $P D^{\prime} \subset D^{\prime}$. Then

(a) If $M$ is a maximal ideal of $D^{\prime}$ and if $P=M \cap D$, then $D_{P}=D_{M}^{\prime}$ and $M=P D_{P} \cap D^{\prime}$. Therefore $D^{\prime}$ is almost Dedekind.

(b) If $D$ is Dedekind, so is $D^{\prime}$.

(c) For $P$ a proper prime ideal of $D, P \in \Delta$ if and only if $D_{P} \supseteq D^{\prime}$. Further, $D^{\prime}=\bigcap_{P \in \Delta} D_{P}$.

(d) If $A^{\prime}$ is an ideal of $D^{\prime}$ and if $A=A^{\prime} \cap D$, then $A^{\prime}=A D^{\prime}$.

(e) $\left\{P D^{\prime}\right\}_{P \in \Delta}$ is the set of proper prime ideals of $D^{\prime}$.

Proof. In (a), we know $P$ is a maximal ideal of $D$. If $N=D-P$, then $D_{P}=D_{N} \subseteq D_{N}^{\prime} \subseteq D_{M}^{\prime} \subset K$. Because $D_{P}$ is a maximal subring of $K, D_{P}=D_{M}^{\prime} . D_{M}^{\prime}$ is therefore a discrete valuation ring with maximal ideal $M D_{M}^{\prime}=P D_{P}$. It follows that $D^{\prime}$ is almost Dedekind and that $M=M D_{M}^{\prime} \cap D^{\prime}=P D_{P} \cap D^{\prime}$.

We now suppose that $D$ is Dedekind. $D^{\prime}$ is almost Dedekind by (a). To show $D^{\prime}$ is Dedekind it suffices, in view of Theorem 3, to show that if $A^{\prime}$ is a nonzero proper ideal of $D^{\prime}, A^{\prime}$ is contained in only finitely many maximal ideals. If now $P_{1}, \cdots, P_{t}$ are the maximal ideals of $D$ which contain $A=A^{\prime} \cap D$, then from assertion (a) we see that $P_{1} D_{P_{1}} \cap D^{\prime}, \cdots, P_{t} D_{P_{t}} \cap D^{\prime}$ are all the maximal ideals of $D^{\prime}$ containing $A^{\prime}$. Hence $D^{\prime}$ is also Dedekind.

To prove (c), we see easily that if $D_{P} \supseteq D^{\prime}$, then $P D^{\prime} \subseteq P D_{P} \subset D_{P}$ so that $P D^{\prime} \subset D^{\prime}$. Conversely, if $P \neq(0)$ and $P D^{\prime} \subset D^{\prime}$, we let $M$ be a maximal ideal of $D^{\prime}$ containing $P D^{\prime}$. Then $P \subseteq P D^{\prime} \cap D \subseteq M \cap D \subset D$ so that $P=M \cap D$ since $P$ is a maximal ideal of $D$. From part (a) we see that $D_{P}=D_{M}^{\prime} \supset D^{\prime}$. This proves $P \in \Delta$ if and only if $D_{P} \supseteq D^{\prime}$. Now if $\left\{M_{\lambda}\right\}_{\lambda \in \Delta}$ is the collection of maximal ideals of $D^{\prime}$, then $D^{\prime}=\cap D_{M_{\lambda}}^{\prime}$ $\left[5\right.$, p. 94]. By (a) and the first part of (c), for each $\lambda, D_{M_{\lambda}}^{\prime}=D_{\left(M_{\lambda} \cap D\right)}$ and $\left(M_{\lambda} \cap D\right) \in \Delta$. It follows that $D^{\prime}=\bigcap_{P \in \Delta} D_{P}$.

In (d) we need only consider the case when (0) $\subset A^{\prime} \subset D^{\prime}$. We shall first show that if $M$ is a maximal ideal of $D^{\prime}$ and if $P=M \cap D$, then $P^{k}=M^{k} \cap D$ for each positive integer $k$. This is true since

$$
\begin{aligned}
P^{k} & =P^{k} D_{P} \cap D=M^{k} D_{M}^{\prime} \cap D=M^{k} D_{M}^{\prime} \cap\left(D^{\prime} \cap D\right) \\
& =\left(M^{k} D_{M}^{\prime} \cap D^{\prime}\right) \cap D=M^{k} \cap D .
\end{aligned}
$$

It is clear that $A D^{\prime} \subseteq A^{\prime}$ and hence, that $f_{\lambda}\left(A D^{\prime}\right) \geqq f_{\lambda}\left(A^{\prime}\right)$ for each $\lambda \in \Lambda$. Since $B=\bigcap_{\lambda \in \Lambda} M_{\lambda}^{\gamma^{\lambda(B)}}$ for each nonzero ideal $B$ of $D^{\prime}$, to show $A D^{\prime}=A^{\prime}$, it suffices to show $f_{\lambda}\left(A D^{\prime}\right)=f_{\lambda}\left(A^{\prime}\right)$ for each $\lambda \in \Lambda$. From part (a) this means we must show that if the integer $k$ is such that $A^{\prime} \subseteq M_{\lambda}^{k}, A^{\prime} \Phi M_{\lambda}^{k+1}$, then $A D^{\prime} \Phi M_{\lambda}^{k+1}$. Because $M_{\lambda}^{k+1} \cap D=P_{\lambda}^{k+1}$ 
where $P_{\lambda}=M_{\lambda} \cap D$, it is sufficient to show $A \nsubseteq P_{\lambda}^{k+1}$. This follows essentially from (iii) of Theorem 2 .

We have $P_{\lambda}^{2}=M_{\lambda}^{2} \cap D \subset P_{\lambda}=M_{\lambda} \cap D$ so that if $m_{\lambda} \in P_{\lambda}-P_{\lambda}^{2}$, then $m_{\lambda} \in M_{\lambda}-M_{\lambda}^{2}$. If now $\xi \in A^{\prime}-M_{\lambda}^{k+1}$, then $s_{\lambda}(\xi)=k$ and $\xi=u m_{\lambda}^{k} / v$ for some $u, v \in D-P_{\lambda}$. Then $v \xi=u m_{\lambda}^{k} \in A-P_{\lambda}^{k+1}$ so that $A \Phi P_{\lambda}^{k+1}$ and our proof of $(d)$ is complete.

By (d), every proper prime ideal of $D^{\prime}$ is of the form $P D^{\prime}$ for some $P \in \Delta$. If, however, $P \in \Delta$, then by (c), $D_{P} \supseteq D^{\prime}$ so that $P D_{P} \cap D^{\prime}$ is a prime ideal of $D^{\prime}$. By (d), $P D_{P} \cap D^{\prime}=\left[\left(P D_{P} \cap D^{\prime}\right) \cap D\right] D^{\prime}=P D^{\prime}$ and consequently, $P D^{\prime}$ is a prime ideal of $D^{\prime}$ for $P \in \Delta$.

Another result concerning almost Dedekind domains which is the analogue of a theorem concerning Dedekind domains is the following corollary. The proof uses results in [4, pp. 257, 261, 281].

Corollary 4. If the integral domain $D$ is almost Dedekind, if $K$ is the quotient field of $D$, and if $D^{\prime}$ is the integral closure of $D$ in a finite algebraic extension field $L$ of $K$, then $D^{\prime}$ is almost Dedekind.

Proof. If $M$ is a nonzero proper prime ideal of $D^{\prime}$, then $P=M \cap D$ is a nonzero proper prime ideal of $D$. If $N=D-P$, then $D_{P}=D_{N}$ and $D_{N}^{\prime}$ is integral over $D_{N}$. Because $D^{\prime}$ is integrally closed in $L, D_{N}^{\prime}$ is also integrally closed in $L$. Thus $D_{N}^{\prime}$ is the integral closure of the discrete valuation ring $D_{N}$ in $L$. Consequently, $D_{N}^{\prime}$ is Dedekind so that $D_{M}^{\prime}=\left(D_{N}^{\prime}\right)_{M D_{N}^{\prime}}$ is a discrete valuation ring. Therefore, $D$ is almost Dedekind.

REMARKs. Corollary 4, as well as the following result, was communicated to us by H. S. Butts and R. C. Phillips.

An integral domain $J$ with unit is almost Dedekind if and only if each ideal of $J$ with prime radical is a prime power.

In a paper, The cancellation law for ideals in a commutative ring, to appear in the Canadian Journal of Mathematics, we show that an integral domain $J$ with unit is almost Dedekind if and only if the cancellation law for ideals is valid in $J$-that is, if $A, B$, and $C$ are ideals of $J$ such that $A B=A C$ and $A \neq(0)$, then $B=C$.

Parts (c), (d), and (e) of Theorem 4 are known when $D$ is a Dedekind domain, though we are unaware of a proof in the literature. For $D$ Dedekind, it is also true that if $\Delta_{1}$ and $\Delta_{2}$ are distinct collections of prime ideals of $D$, then $\bigcap_{P \in \Delta_{1}} D_{P} \neq \bigcap_{P \in \Delta_{2}} D_{P}$. We believe this assertion is false for $D$ almost Dedekind, but lack an example.

The definition of an almost Dedekind domain can easily be extended to the case of an integral domain without unit. Theorem 1 is no longer valid in this case. For example, if $A$ is any ideal of a Dedekind domain $D$, then for each prime ideal $P$ of the domain $A, A_{P}$ is a discrete valuation ring. 


\section{REFERENCES}

1. I. S. Cohen, Commutative rings with restricted minimum conditions, Duke Math. J. 17 (1950), 27-41.

2. S. MacLane and O. F. G. Schilling, Infinite number fields with Noether ideal theories, Amer. J. Math. 61 (1939), 771-782.

3. N. Nakano, Idealtheorie in einem speziellen unendlichen algebraischen Zahlkorper, J. Sci. Hiroshima Univ. 16(1953), 425-439.

4. O. Zariski and P. Samuel, Commutative algebra. I, Van Nostrand, Princeton, N. J., 1953.

5. —-, Commutative algebra. II, Van Nostrand, Princeton, N. J., 1960.

Louisiana State University and

UNIVERSITY OF WISCONSIN

\section{A NOTE ON PERTURBATION THEORY FOR SEMI-GROUPS OF OPERATORS}

\section{A. OLUBUMMO}

1. Introduction. Let $X$ be a Banach lattice and $\Sigma=\{T(\xi) ; \xi \geqq 0\}$ a family of bounded operators on $X$ satisfying

(i) $T\left(\xi_{1}+\xi_{2}\right)=T\left(\xi_{1}\right) T\left(\xi_{2}\right), T(0)=I, 0 \leqq \xi_{1}, \xi_{2}<\infty$;

(ii) $x \geqq 0$ implies that $T(\xi) x \geqq 0$ for each $\xi \geqq 0$;

(iii) $\|T(\xi)\| \leqq 1$ for $\xi \geqq 0$;

(iv) $\lim _{\xi \downarrow 0} T(\xi) x=x$ for each $x \in X$.

We shall refer to $\Sigma$ as a strongly continuous semi-group of positive contraction operators.

Lumer [2] has shown that for a complex (real) normed linear space $X$, there exists at least one complex-valued (real-valued) function $[x, y]$ called a semi-inner-product defined on $X \times X$ having the following properties:

$$
\begin{aligned}
{[x+y, z] } & =[x, z]+[y, z], \\
{[\lambda x, z] } & =\lambda[x, z], \\
{[x, x] } & =\|x\|^{2} \\
|[x, z]| & \leqq\|x\|\|z\| .
\end{aligned}
$$

If $X$ is a Banach lattice, then Phillips [4] has shown that there exists a semi-inner-product with the further properties:

Received by the editors April 15, 1963. 